\title{
Genome Size: A Novel Predictor of Nut Weight and Nut Size of Walnut Trees
}

\author{
Saadat Sarikhani Khorami ${ }^{1}$ and Kazem Arzani ${ }^{1}$ \\ Department of Horticultural Science, Tarbiat Modares University, Tehran, \\ Iran \\ Ghasem Karimzadeh \\ Department of Plant Breeding, Tarbiat Modares University, Tehran, Iran
}

Abdolali Shojaeiyan
Department of Horticultural Science, Tarbiat Modares University, Tehran,
Iran

Wilco Ligterink

Wageningen Seed Lab, Laboratory of Plant Physiology, Wageningen University, The Netherlands

Additional index words. flow cytometry, $2 C_{x}$ DNA value, lateral bearing, nut weight, linear regression

\begin{abstract}
Plant genetic diversity is the fundamental of plant-breeding programs to improve desirable characteristics. Hence, evaluation of genetic diversity is the first step in fruit-breeding programs. Accordingly, the current study was carried out to evaluate 25 superior walnut genotypes in respect of phenotypic and cytological characteristics. For this purpose, 560 walnut genotypes in southwest of Iran were evaluated based on UPOV and International Plant Genetic Resources Institute (IPGRI) descriptor. After a 2-year primary evaluation, 25 superior genotypes were selected for future phenotypic and genome size assessment. Flow cytometry was used to estimate genome size of the selected superior genotypes. A high genetic diversity was found in walnut population collected from the southwest of Iran. The selected superior genotypes had high yield, lateral bearing, thin-shell thickness $(0.90-1.64 \mathrm{~mm})$, high nut $(12.54-19.80 \mathrm{~g})$ and kernel $(7.02$ $9.91 \mathrm{~g}$ ) weight with light (L) to extra light (EL) kernel color which easily can be removed from the shell. Also, FaBaCh2 genotype turned out to be protogynous being important as a pollinizer cultivar. In addition to extensive phenotypic analysis, genome size was determined. The studied genotypes were diploid $(2 n=2 x=32)$ and varied in genome size from 1.29 (FaBaAv2) to $1.40 \mathrm{pg}$ (FaBaNs12). Correlation analysis showed that lateral bearing, budbreak date, nut size, and weight were the main variables contributing to walnut production. A linear relationship was found between genome size and nut weight $\left(r=0.527^{* *}\right)$, kernel weight $\left(r=0.551^{* *}\right)$, and nut size index (NSI) $(r=0.487 * *)$. Therefore, genome size can be considered as a strong and valuable tool to predict nut and kernel weight and nut size.
\end{abstract}

Plant genetic diversity provides opportunity for breeders to release new cultivars during plant-breeding programs (Govindaraj et al., 2015). However, many plants, such as fruit trees, have a long breeding cycle. Long breeding cycle of fruit trees specifically nut crops is the major constraint for breeders (van Nocker and Gardiner, 2014). One of the basic strategies for reducing the length of breeding cycle is the introduction of superior genotypes. Moreover, germplasm screening of fruit trees in origin and diversity centers is also considered as basic and applied strategies

Received for publication 20 Nov. 2017. Accepted for publication 15 Jan. 2018.

We would like to thank Tarbiat Modares University (TMU) for providing facilities and financial support. We also thank Narges Firoozi for her kind collaboration in genome size assessment.

${ }^{1}$ Corresponding authors. E-mail: arzani_k@modares. ac.ir or saadat.sarikhani@modares.ac.ir. for reducing the length of breeding cycle and for introducing new cultivars (Arzani et al., 2008; van Nocker and Gardiner, 2014).

Iran, as the third largest walnut producer (with more than $445,829 t$ ), is one of the main origin and diversity centers of walnut around the world (FAO, 2014). Because of being an origin center and seed propagation and openpollination of walnut, high genetic diversity for walnut has been reported in different regions of the country (Khadivi-Khub et al., 2015). Hence, germplasm evaluation is the first and fundamental step in walnut breeding programs (Arzani et al., 2008).

Genetic diversity has been evaluated in different aspects such as phenotypic, biochemical, and molecular traits (Govindaraj et al., 2015). Walnut genetic diversity is routinely evaluated with the help of phenotypic and biochemical methods to obtain valuable information from the gene pool and identify superior genotypes with desirable characterization. In addition to nucleotide sequences, genetic diversity also refers to variation in the chromosomes and genome size (Bhat and Kudesia, 2011). Therefore, cytological studies can be powerful tools to evaluate walnut genetic diversity. Among cytological traits, nuclear DNA content ( $C$-value) is a key characteristic in plant breeding and in evaluating germplasm (Abedi et al., 2015; Doležel and Bartos, 2005; Doležel et al., 2007; Greilhuber et al., 2005; Karimzadeh et al., 2010; Karimzadeh et al., 2011; Knight et al., 2005; Mahdavi and Karimzadeh, 2010). The term $C$-value was first described by Swift (1950) as the DNA amount of an unreplicated haploid chromosome complement i.e., a gamete (Greilhuber et al., 2005). Monoploid genome size (the amount of DNA of one chromosome set, $1 C_{x}$ value, with chromosome base number $x$ ) and holoploid genome size (the amount of DNA of the whole chromosome complement, $1 C$-value, with chromosome number $n$, irrespective of the degree of generative polyploidy and aneuploidies etc.) are explained in detail by Greilhuber et al. (2005). Furthermore, analytical tools such as flow cytometry are a reliable and convenient method to estimate genome size of plant communities such as ornamental plants (Abedi et al., 2015), medicinal plants (Javadian et al., 2017; Mahdavi and Karimzadeh, 2010; Majdi et al., 2010; Sadat Noori et al., 2017; Tarkesh Esfahani et al., 2016; Tavan et al., 2015) and fruit trees such as peach (Baird et al., 1994), olive (Brito et al., 2008; Loureiro et al., 2007b), coconut (Freitas et al., 2015), and Malus species (Hofer and Meister, 2010; Korban et al., 2009; Podwyszyńska et al., 2016). Unlike other fruit trees and plant species, there has been no study on the genome size of different walnut genotypes and cultivars, only flow cytometry is used to determine the ploidy level of walnut samples in in vitro conditions with the aim of identifying haploid plants (Sadat Hosseini Grouh et al., 2011).

Today, analysis of genome size as a genetic parameter is very convenient, therefore, study of genome size and its correlation with important traits can be consider as useful tools in walnut breeding programs and germplasm evaluation. Because of this fact, the aims of the current research were to evaluate the morpho-cytological diversity of some superior and promising walnut genotypes in the southwest of Iran and determine the genome size of the selected superior genotypes. In addition, the relationship between genome size and some important phenotypic traits were investigated.

\section{Materials and Methods}

Plant materials. Fars province is the main walnut producing area in the southwest of Iran. Five hundred and sixty walnut genotypes from Bavanat $(\mathrm{FaBa})$ and Eqlid $(\mathrm{FaEq})$ regions of Fars were used in this research. After a 2-year primary evaluation (2010-12), 25 superior genotypes were selected for detailed phenotypic and cytological assessment. Superior genotypes were selected based on phenotypic data and were superior 
in terms of some important breeding traits such as nut and kernel weight and kernel percentage, respectively, more than $13 \mathrm{~g}, 6.5$ $\mathrm{g}$, and $50 \%$, thin shell, L- EL kernel color, and easy removal in halves (Akca and Ozongun, 2004; IPGRI, 1994; Sharma and Sharma, 2001b; Simsek et al., 2010; Zeneli et al., 2005).

Phenotypic traits. Selected superior genotypes were morphologically assessed for 3 years (2013-15). For this purpose, 14 important traits were evaluated based on International Plant Genetic Resources Institute (IPGRI) descriptors (IPGRI, 1994). Phenological data such as budbreak date, the first and last pollen-shedding date, the first and last pollen reception date and harvesting date were evaluated by days after reference standards (DARS). The earliest leafing genotype (15th of March for budbreak and flowering date and first of September for harvesting date) was considered as a reference standard (Arzani et al., 2008). Twenty nuts of each selected genotype were used to study pomological traits (IPGRI, 1994). Nuts were harvested when the green outer hull splitted and the nut exposed. After harvesting and removing hulls, nuts (walnut seeds) were kept in the shade at room temperature for 1 month (Zeneli et al., 2005). Nut and kernel weight were determined, using a digital balance. Kernel percentage was calculated by kernel weight/ nut weight $\times 100$. Lateral-bearing habit was measured based on the percentage of current season lateral shoots with fruitlets (Arzani et al., 2008). Nut-size index, as a parameter of nut size, was determined by the average of nut length, nut width, and nut thickness. Nut size and shell thickness were measured using a digital caliper. Yield and kernel color were determined based on IPGRI descriptors (IPGRI, 1994).

Genome size assessment. Flow cytometry was used to estimate the genome size $\left(2 C_{x}\right.$ DNA value) of each genotype using the propidium iodide (PI) staining method. To extract the plant cells' nuclei, $1 \mathrm{~cm}^{2}$ pieces of young and well-developed leaves of each superior genotype with the same mass of the internal reference standard (Solanum lycopericum cv. Stupicke, 2C DNA $=1.96$ pg) were used. For this purpose, the leaves of walnut and internal standard were chopped with a sharp razor blade in $1 \mathrm{~mL}$ woody plant buffer (Loureiro et al., 2007a). The suspension of isolated nuclei was filtered through Partec (Munster, Germany) 50 and $30 \mu \mathrm{m}$ nylon mesh filters to remove cell debris. The nuclear suspension of each sample was treated with $50 \mu \mathrm{g} \cdot \mathrm{mL}^{-1}$ RNase to prevent staining of double-stranded RNA and stained with $50 \mu \mathrm{g} \cdot \mathrm{mL}^{-1}$ PI (Fluka) (Javadian et al., 2017; Sadat Noori et al., 2017; Tarkesh Esfahani et al., 2016; Tavan et al., 2015). The nuclei suspension was analyzed with a BD FACSCanto II flow cytometer (BD Biosciences, Bedford, MA), using BD FACSDiva software. Subsequently, data were transferred to a Flowing Software version 2.5.0 (Cell Imaging Core; Turku Center for Biotechnology, Turku, Finland) to be editable in Partec FloMax ver. $2.4 \mathrm{e}$ (Partec). The measurements of relative fluorescence intensity of stained nuclei were performed on a linear scale. At least 5000 nuclei were analyzed for each sample. The absolute DNA amount of a sample was calculated based on the values of the G1 peak means (Doležel and Bartos, 2005; Doležel et al., 2007) as follows:

$$
\begin{gathered}
\text { Sample } 2 C_{x} \mathrm{DNA}(\mathrm{pg}) \\
=(\text { Sample G1 peak mean } / \\
\quad \text { Standard G1 peak mean }) \\
\times \text { Standard } 2 C \text { DNA }(\mathrm{pg}) .
\end{gathered}
$$

Monoploid genome size in bps was calculated based on a converting formula proposed by Doležel et al. (2003), where 1 pg of DNA represents 978 mega bps (Mbp).

Data Analyses. As mentioned previously, phenotypic characteristics were evaluated for 3 years. The obtained data were analyzed using SAS (SAS Institute, Cary, NC). Oneway analysis of variance was carried out to determine significant differences between phenotypic and genome size data. Genome size and some high-heritable phenotypic traits such as nut and kernel weight, kernel percentage, lateral bearing, budbreak date, pollen-shedding, and reception date and harvesting date were used for principal component and cluster analysis. Before principal component analysis (PCA), cluster and bivariate correlation analysis, the data were normalized by a specific reference sample. A heat map was made with Spearman correlation coefficient matrices R-packages "MASS," "Hmisc," "VGAM," and "gplots." PCA analysis was performed using Minitab software (Ver. 17). Cluster analysis was conducted using Ward method and Minitab software (Ver. 17). Furthermore, linear regression analysis was carried out to find relationship between $2 C_{x}$ DNA values and some phenotypic traits (nut and kernel weight and NSI), using SPSS version 22

\section{Results}

Phenotypic traits. Twenty five superior genotypes were selected based on a primary evaluation of 560 genotypes in the southwest of Iran. Significant differences were observed between these genotypes in respect to phenotypic traits. Nut and kernel weight ranged from 12.58 to $19.80 \mathrm{~g}$ and 7.11 to $9.91 \mathrm{~g}$, respectively (Table 1). Genotype FaEgFm1 had the highest nut and kernel weight. The highest and lowest kernel percentages were observed in FaBaHr32 (61.23\%) and FaEqAg1 (48.37\%) genotypes, respectively. By contrast, $\mathrm{FaBaHr} 32$ and $\mathrm{FaEqAg} 1$ genotypes had the smallest and largest nut size among the studied genotypes. Nut size index of FaBaHr32 and FaEqAg1 genotypes were 31.35 and 42.47, respectively (Table 1 ). Shell thickness varied between 0.90 and $1.64 \mathrm{~mm}$. All superior genotypes, except FaEqFm1 and $\mathrm{FaEqHm} 2$, were classified as nut with paper shell, because their shell thickness was less than $1.50 \mathrm{~mm}$ (IPGRI, 1994). A wide variation was observed between the studied genotypes especially in respect to lateral bearing. In contrast to commercial cultivars, bearing habit of old walnut trees is mostly lateral. Lateral bearing is one of the main components determining the yield of walnut trees (Sarikhani Khorami et al., 2014). Therefore, the most important aim in Iranian walnutbreeding programs is to increase lateralbearing percentage and find traits with high correlation with lateral bearing (KhadiviKhub et al., 2015). In the current study, lateral bearing of superior genotypes varied between $54 \%$ and $94.47 \%$, with lateral bearing of $\mathrm{FaBaKr} 2, \mathrm{FaBaCh} 2$ and $\mathrm{FaEqNs} 9$ being more than $80 \%$ (Table 1 ).

Although, the occurrence of phenological traits such as leafing and harvest date in different genotypes relative to each other depends on environmental conditions (Arzani et al., 2008), but phenological traits are highheritability traits (Hansche et al., 1972; Ramos, 1997). Therefore, evaluation of phenological traits such as other high-heritability traits can be considered as a useful tool to study walnut germplasm (Arzani et al., 2008). The results showed that FaBaAg1 (25 DARS) and FaBaAv2 (21 DARS) were late-leafing and midseason ripening genotypes (Table 2). In terms of harvesting date, all studied genotypes were early and midseason repining genotypes. The average length of pollen shedding and pistil receptivity were 15 and $8 \mathrm{~d}$, respectively. Genotype FaEqAh1 had the longest period of pollen shedding $(20 \mathrm{~d})$. In addition, all studied genotypes had high to very high bearing with $\mathrm{L}$ to EL kernel color (Table 2). In walnut, dichogamy is one of the main traits that can hamper high productivity. Dichogamy refers to the overlap between the period of pistil receptivity and pollen shedding and there are two modes of dichogamy among walnut trees: protandry and protogyny. Protandry is the most common mode of dichogamy in walnut (Arzani et al., 2008; Vahdati et al., 2014). Some walnut genotypes are homogamous with a period of pistil receptivity and pollen shedding that overlaps at least $6 \mathrm{~d}$ (Arzani et al., 2008; Yarilgac et al., 2001). Accordingly, FaBaHr13, FaBaNs12, $\mathrm{FaBaAg} 1$, and FaEqAal genotypes can be considered homogamous. Except $\mathrm{FaBaCh} 2$, all other studied genotypes were protandrous (Table 2).

Genome size. Flow cytometric histograms of nuclear DNA amounts of 25 studied superior genotypes are presented in Fig. 1. The left peaks refer to G1 of walnut samples and the right peaks to G1 of the Solanum lycopersicum cv. Stupicke ( $2 C$ DNA $=1.96$ pg) as a reference standard (Fig. 1). There was significant difference among walnut superior genotypes in terms of $2 C_{x}$ DNA amount. The results showed that all superior genotypes were diploid $(2 n=2 x=32)$ and their $2 C_{x}$ DNA amount varied from $1.29 \mathrm{pg}$ in genotypes FaBaAv2 and FaBaNs19 to $1.42 \mathrm{pg}$ in genotype FaEqFm1 (Table 3). After $\mathrm{FaEqFm} 1$, genotypes $\mathrm{FaBaNs} 12$ and FaBaAg1 had the highest amount of nuclear DNA amounts. Furthermore, the average amount of walnut $2 C_{x}$ genome size was $1315 \mathrm{Mbp}$ [monoploid genome size $\left(1 C_{x^{-}}\right.$ DNA) $=657.80 \mathrm{Mbp}]$. On the other hand, 
Table 1. The average of quantitative phenotypic traits of superior walnut genotypes in southwest Iran during 2013-15.

\begin{tabular}{|c|c|c|c|c|c|c|}
\hline Genotype & Nut wt (g) & Kernel wt (g) & Kernel percentage (\%) & Nut-size index & Shell thickness (mm) & Lateral bearing $(\%)$ \\
\hline FaBaHr32 & $12.54 \pm 0.09$ & $7.67 \pm 0.11$ & $61.23 \pm 0.85$ & $31.35 \pm 0.07$ & $0.90 \pm 0.02$ & $59.78 \pm 0.20$ \\
\hline $\mathrm{FaBaHr} 42$ & $13.64 \pm 0.15$ & $7.37 \pm 0.13$ & $54.07 \pm 0.88$ & $32.48 \pm 0.09$ & $1.02 \pm 0.02$ & $69.40 \pm 0.24$ \\
\hline FaBaNs 12 & $18.18 \pm 0.20$ & $9.04 \pm 0.08$ & $49.76 \pm 0.41$ & $38.61 \pm 0.09$ & $1.33 \pm 0.03$ & $78.73 \pm 0.20$ \\
\hline FaBaNs19 & $13.49 \pm 0.13$ & $7.71 \pm 0.11$ & $57.25 \pm 0.98$ & $32.65 \pm 0.12$ & $1.18 \pm 0.02$ & $73.60 \pm 0.29$ \\
\hline FaBaNs36 & $15.19 \pm 0.28$ & $8.16 \pm 0.12$ & $53.77 \pm 0.49$ & $33.61 \pm 0.09$ & $1.40 \pm 0.01$ & $57.29 \pm 0.29$ \\
\hline FaBaNs39 & $14.62 \pm 0.13$ & $7.68 \pm 0.07$ & $52.56 \pm 0.40$ & $35.29 \pm 0.05$ & $1.38 \pm 0.02$ & $58.82 \pm 0.44$ \\
\hline FaBaKr2 & $16.16 \pm 0.16$ & $8.36 \pm 0.08$ & $51.77 \pm 0.46$ & $36.33 \pm 0.12$ & $1.50 \pm 0.02$ & $94.47 \pm 0.57$ \\
\hline FaBaAv2 & $13.72 \pm 0.13$ & $7.02 \pm 0.07$ & $51.19 \pm 0.46$ & $35.00 \pm 0.08$ & $1.39 \pm 0.02$ & $54.47 \pm 0.50$ \\
\hline FaBaMm1 & $16.57 \pm 0.14$ & $8.51 \pm 0.09$ & $51.37 \pm 0.30$ & $33.82 \pm 0.09$ & $1.48 \pm 0.02$ & $66.53 \pm 0.32$ \\
\hline $\mathrm{FaBaGm} 2$ & $14.00 \pm 0.27$ & $7.51 \pm 0.16$ & $53.69 \pm 0.67$ & $35.91 \pm 0.12$ & $1.36 \pm 0.02$ & $55.73 \pm 0.38$ \\
\hline $\mathrm{FaBaCh} 2$ & $13.67 \pm 0.11$ & $8.28 \pm 0.06$ & $60.58 \pm 0.45$ & $34.91 \pm 0.08$ & $1.10 \pm 0.02$ & $87.62 \pm 0.49$ \\
\hline FaBaAg1 & $14.96 \pm 0.08$ & $7.64 \pm 0.12$ & $51.05 \pm 0.72$ & $36.73 \pm 0.05$ & $1.24 \pm 0.01$ & $58.90 \pm 0.47$ \\
\hline FaBaHr13 & $15.81 \pm 0.13$ & $8.47 \pm 0.11$ & $53.58 \pm 0.50$ & $38.24 \pm 0.17$ & $0.99 \pm 0.01$ & $54.00 \pm 0.39$ \\
\hline FaBaAs32 & $15.54 \pm 0.14$ & $8.24 \pm 0.05$ & $53.09 \pm 0.61$ & $37.95 \pm 0.10$ & $1.43 \pm 0.02$ & $67.43 \pm 0.53$ \\
\hline $\mathrm{FaBaHm} 5$ & $15.22 \pm 0.11$ & $8.23 \pm 0.07$ & $54.05 \pm 0.41$ & $36.29 \pm 0.06$ & $1.15 \pm 0.01$ & $67.57 \pm 0.28$ \\
\hline $\mathrm{FaBaCh} 4$ & $14.62 \pm 0.09$ & $8.08 \pm 0.08$ & $55.22 \pm 0.44$ & $35.16 \pm 0.05$ & $1.30 \pm 0.03$ & $76.70 \pm 0.35$ \\
\hline FaEqNs5 & $14.48 \pm 0.08$ & $7.18 \pm 0.08$ & $49.59 \pm 0.27$ & $34.87 \pm 1.05$ & $1.10 \pm 0.03$ & $75.00 \pm 2.00$ \\
\hline FaEqNs9 & $13.47 \pm 0.08$ & $7.51 \pm 0.01$ & $55.76 \pm 0.27$ & $35.68 \pm 0.10$ & $1.10 \pm 0.03$ & $80.00 \pm 1.26$ \\
\hline FaEqFm1 & $19.80 \pm 0.05$ & $9.91 \pm 0.05$ & $50.05 \pm 0.36$ & $39.06 \pm 0.37$ & $1.64 \pm 0.04$ & $75.00 \pm 0.72$ \\
\hline FaEqAa1 & $15.10 \pm 0.12$ & $8.86 \pm 0.12$ & $58.69 \pm 1.19$ & $35.95 \pm 0.30$ & $1.38 \pm 0.05$ & $62.50 \pm 1.61$ \\
\hline FaEqDm 1 & $14.19 \pm 0.05$ & $7.89 \pm 0.15$ & $55.60 \pm 0.89$ & $35.90 \pm 0.33$ & $1.22 \pm 0.03$ & $65.00 \pm 0.58$ \\
\hline FaEqHm1 & $13.41 \pm 0.03$ & $8.04 \pm 0.07$ & $59.96 \pm 0.61$ & $35.01 \pm 0.20$ & $1.30 \pm 0.03$ & $65.00 \pm 1.00$ \\
\hline $\mathrm{FaEqHm} 2$ & $15.95 \pm 0.05$ & $8.40 \pm 0.12$ & $52.66 \pm 0.62$ & $37.50 \pm 0.62$ & $1.56 \pm 0.02$ & $75.00 \pm 1.53$ \\
\hline FaEqAh1 & $13.08 \pm 0.06$ & $7.58 \pm 0.19$ & $57.95 \pm 1.36$ & $33.94 \pm 0.40$ & $0.95 \pm 0.03$ & $72.00 \pm 1.32$ \\
\hline FaEqAg1 & $19.02 \pm 0.08$ & $9.20 \pm 0.07$ & $48.37 \pm 0.41$ & $42.47 \pm 0.48$ & $0.90 \pm 0.02$ & $72.50 \pm 0.93$ \\
\hline $\operatorname{LSD}(0.01)$ & 0.53 & 0.62 & 3.37 & 1.34 & 0.09 & 2.66 \\
\hline
\end{tabular}

Table 2. The average of qualitative phenotypic traits of superior walnut genotypes in southwest Iran during 2013-15.

\begin{tabular}{|c|c|c|c|c|c|c|c|c|}
\hline \multirow{2}{*}{ Genotype } & \multirow{2}{*}{ Kernel color } & \multirow{2}{*}{ Bearing $^{z}$} & \multicolumn{6}{|c|}{ Phenological dates $\left(\mathrm{DARS}^{\mathrm{y}}\right)$} \\
\hline & & & Budbreak & Harvest & \multicolumn{2}{|c|}{ Male bloom } & \multicolumn{2}{|c|}{ Female bloom } \\
\hline FaBaHr32 & $\mathrm{L}$ & 7 & 7 & 12 & 14 & 27 & 25 & 32 \\
\hline FaBaNs 12 & EL & 9 & 8 & 15 & 23 & 36 & 29 & 36 \\
\hline FaBaNs19 & $\mathrm{L}$ & 8 & 5 & 12 & 15 & 28 & 32 & 39 \\
\hline FaBaNs36 & $\mathrm{L}$ & 7 & 5 & 8 & 10 & 25 & 27 & 34 \\
\hline FaBaNs39 & $\mathrm{L}$ & 7 & 5 & 7 & 21 & 35 & 31 & 38 \\
\hline FaBaMm 1 & EL & 9 & 14 & 10 & 21 & 36 & 39 & 47 \\
\hline $\mathrm{FaBaGm} 2$ & $\mathrm{~L}$ & 7 & 11 & 12 & 14 & 29 & 34 & 42 \\
\hline $\mathrm{FaBaCh} 2$ & $\mathrm{~L}$ & 9 & 9 & 6 & 20 & 34 & 14 & 22 \\
\hline FaBaAg1 & EL & 9 & 25 & 19 & 43 & 58 & 45 & 52 \\
\hline FaBaHr13 & EL & 7 & 8 & 8 & 24 & 39 & 31 & 38 \\
\hline FaBaAs32 & $\mathrm{L}$ & 8 & 11 & 7 & 14 & 27 & 27 & 35 \\
\hline $\mathrm{FaBaHm} 5$ & EL & 8 & 15 & 17 & 23 & 37 & 40 & 48 \\
\hline FaEqAa1 & $\mathrm{L}$ & 7 & 6 & 12 & 10 & 26 & 20 & 27 \\
\hline FaEqDm 1 & EL & 8 & 5 & 11 & 23 & 38 & 31 & 39 \\
\hline $\mathrm{FaEqHm} 1$ & $\mathrm{~L}$ & 7 & 4 & 10 & 7 & 24 & 28 & 37 \\
\hline $\mathrm{FaEqHm} 2$ & $\mathrm{~L}$ & 8 & 8 & 15 & 16 & 33 & 30 & 39 \\
\hline FaEqAh1 & EL & 7 & 4 & 13 & 6 & 26 & 25 & 34 \\
\hline FaEqAg1 & EL & 9 & 10 & 14 & 15 & 33 & 33 & 42 \\
\hline
\end{tabular}

${ }^{\mathrm{z}}$ Bearing (7: high, 8: high to very high, 9: very high).

${ }^{\mathrm{y}}$ DARS = days after reference standard (Reference standards were 15th of March for budbreak and flowering date and first of September for harvesting date). $\mathrm{L}=$ light, $\mathrm{EL}=$ extra light.

human genome size $(6153 \mathrm{Mbp})$ is $\approx 4.7$ times of walnut genome size (International Human Genome Sequencing Consortium, 2004).

Multivariate analysis. PCA is commonly used to detect and quantify the genetic structure of populations (Ma and Amos 2012). In this study, PCA was performed to determine parameters quota in total variation. The results showed that the first principal component could explain $42.3 \%$ of the variation.
Furthermore, the first and second component explained generally $9 \%$ of variation. The first component was influenced by budbreak date, harvesting date, pollen-shedding and its reception date. Nut and kernel weight and genome size were contributed to second principal component (Fig. 2). As mentioned in the previous section, phenotypic traits and genome size were also used for cluster analysis. Based on cluster analysis, 25 studied genotypes were classified into four groups. Sixteen out of 25 superior genotypes were placed in the same group (group 1) having the least genetic distance (Fig. 2). Genotypes FaBaNs12, FaEqAg1 and FaEqFm1 were clustered in group 2 having the highest genome size and nut and kernel weight. $\mathrm{FaBaAg} 1$ and $\mathrm{FaBaAv} 2$ as late-leafing genotypes were classified in cluster group 4 and had least and highest genetic distance with cluster group 3 and 1, respectively (Fig. 2).

To investigate associations between genome size and phenotypic characteristics, 

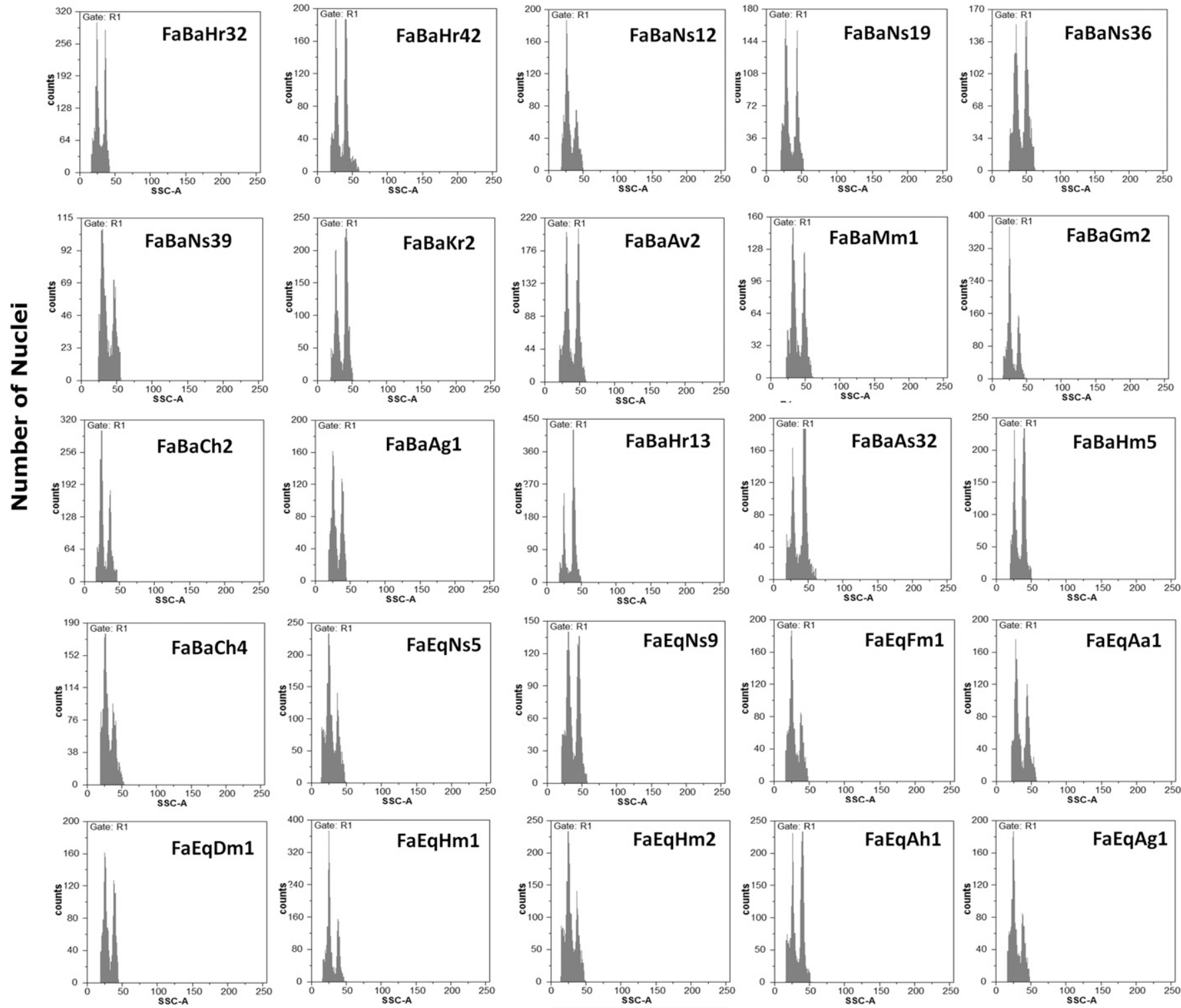

\section{Relative fluorescence intensity}

Fig. 1. Flow cytometric histograms of $2 C_{x}$ DNA content of 25 walnut superior genotypes in southwest Iran (FaBa: Bavanat region and FaEq: Eqlid region): The left peaks refer to G1 of walnut samples and the right peaks refer to G1 of the Solanum lycopersicum cv. Stupicke ( 2 C DNA = $1.96 \mathrm{pg})$ as a reference standard. Mean $\mathrm{CV} \%$ of the peaks was less than $5 \%$.

a correlation matrix was generated by performing spearman correlation analysis across all 25 walnut superior genotypes. A strong and positive correlation was observed between budbreak date, pollen shedding and pistil receptivity $(P<0.01)$ (Fig. 3). Among the positive correlations between the harvesting date and other phenological traits, only the correlation with budbreak date was significant at $P<0.05$. A significant and negative correlation $(P<0.01)$ was observed between several phenological characteristics and kernel percentage. In addition, harvesting date had a significant negative correlation with kernel color. Yield (bearing) is the main objective of breeding programs of fruit trees including walnut (Amiri et al., 2010). A heat map of spearmen correlations between all measured traits, showed that bearing had a positive correlation with budbreak date, lateral bearing $(P<0.01)$, nut and kernel weight and NSI $(P<0.05)$. By contrast, a significant negative correlation was observed between bearing and kernel percentage and kernel color (Fig. 3).

Because of the high heritability, fruit breeders are interested in pomological traits in walnut-breeding programs. However, knowledge of strong associations between pomological traits and other characteristics can guide appropriate selection schemes for walnut-breeding programs (Amiri et al., 2010). In this study, a significant and positive correlation was observed between nut and kernel weight and between both of them and NSI. SI had a negative correlation with kernel percentage and kernel color. A similar correlation was found between nut weight and kernel percentage. The bivariate correlation coefficients between genome size and other studied traits showed that there was no significant correlation between genome size and all phenological and some pomological characteristics (bearing, kernel percentage, shell strength, and lateral bearing). In contrast to phenological traits, genome size had a significant negative correlation $(P<0.01)$ with kernel color. Moreover, we found a strong positive correlation between genome size and nut and kernel weight and SI (Fig. 3). Linear regression analysis showed that these were highly significant with $r=0.527^{* *}$, $0.551^{* *}$, and $0.487^{* *}$, respectively (Fig. 4).

\section{Discussion}

Seedling-originated trees, nature of pollination (heterodichogamy and anemophily) and the fact that Iran is one of the origin centers for walnut are the reasons for a high 
genetic diversity in the walnut population of Iran (Arzani et al., 2008, Karimi et al., 2010; Khadivi-Khub and Ebrahimi, 2015). This native genetic diversity presents many opportunities for walnut breeding (Akca and Ozongun, 2004). Because of high genetic diversity in Iranian walnut population, germplasm evaluation is the fundamental step in walnut-breeding programs (Arzani et al., 2008). Genetic diversity is evaluated by different aspects such as phenotypic, biochemical, cytological, and molecular traits (Govindaraj et al., 2015). Study of phenotypic traits leads to acquisition of valuable information from the germplasm and identification of superior genotypes with desirable characteristics (Bernard et al., 2018). High yield, late leafing, lateral bearing, earlier

Table 3. Genome size and $2 C$ DNA content of the studied walnut superior genotypes in southwest Iran.

\begin{tabular}{lcc}
\hline Genotype & Mean $2 C_{x}$ & $\begin{array}{c}1 C_{x} \text { monoploid } \\
\text { genome size }(\mathrm{Mbp})\end{array}$ \\
\hline FaBaHr32 & $1.32 \pm 0.01$ & 645.48 \\
FaBaHr42 & $1.32 \pm 0.01$ & 645.48 \\
FaBaNs12 & $1.40 \pm 0.01$ & 684.6 \\
FaBaNs19 & $1.29 \pm 0.01$ & 630.81 \\
FaBaNs36 & $1.34 \pm 0.01$ & 655.26 \\
FaBaNs39 & $1.32 \pm 0.01$ & 645.48 \\
FaBaKr2 & $1.33 \pm 0.01$ & 650.37 \\
FaBaAv2 & $1.29 \pm 0.00$ & 630.81 \\
FaBaMm1 & $1.33 \pm 0.01$ & 650.37 \\
FaBaGm2 & $1.32 \pm 0.00$ & 645.48 \\
FaBaCh2 & $1.32 \pm 0.01$ & 645.48 \\
FaBaAg1 & $1.34 \pm 0.00$ & 655.26 \\
FaBaHr13 & $1.36 \pm 0.01$ & 665.04 \\
FaBaAs32 & $1.37 \pm 0.00$ & 669.93 \\
FaBaHm5 & $1.35 \pm 0.01$ & 660.15 \\
FaBaCh4 & $1.35 \pm 0.00$ & 660.15 \\
FaEqNs5 & $1.36 \pm 0.01$ & 665.04 \\
FaEqNs9 & $1.35 \pm 0.00$ & 660.15 \\
FaEqFm1 & $1.42 \pm 0.01$ & 694.38 \\
FaEqAa1 & $1.36 \pm 0.00$ & 665.04 \\
FaEqDm1 & $1.35 \pm 0.00$ & 660.15 \\
FaEqHm1 & $1.37 \pm 0.00$ & 669.93 \\
FaEqHm2 & $1.35 \pm 0.00$ & 660.15 \\
FaEqAh1 & $1.34 \pm 0.00$ & 655.26 \\
FaEqAg1 & $1.38 \pm 0.00$ & 674.82 \\
Average & 1.35 & 657.80 \\
LSD $(0.01)$ & 0.016 & \\
\hline & &
\end{tabular}

fruiting, large nut and high kernel percentage, great kernel quality and compatibility with different ecological conditions are main objectives of walnut-breeding programs (Arzani et al., 2008; Aslantas, 2006; Ebrahimi et al., 2015; Keles et al., 2014). In the current study, 25 walnut superior genotypes were selected based on phenotypic characteristics having the highest yield, nut weight, lateral bearing, kernel percentage, and quality among the evaluated walnut population in the southwest of Iran. Because of the desirable breeding traits, these superior genotypes can be used as parents in future hybridization programs for appearance desirable traits in progeny. In addition, considering that these selected genotypes are superior in many of the desired traits, they can be released as new commercial cultivars in the future walnut-breeding programs (Arzani et al., 2008; Govindaraj et al., 2015).

Phenological traits especially leafing dates are one of the most important aims for new cultivars. Late-leafing genotypes not only can be immune to risks of late spring frost, but also can be cultivated in the mountain areas where late spring frosts are frequent (Akca and Ozongun, 2004). The selected superior genotypes in this study were mid-leafing and late-leafing genotypes. Among them, especially genotypes FaBaAg1 and FaBaAv2 with, respectively, 25 and $21 \mathrm{~d}$ delay compared with the reference standard in respect to leafing, can be considered as promising and valuable genotypes for future breeding programs. Several late-leafing genotypes have been introduced in previous studies (Akca and Ozongun, 2004; Arzani et al., 2008; Ghasemi et al., 2012). However, genotypes $\mathrm{FaBaAg} 1$ and $\mathrm{FaBaAv} 2$ not only are late-leafing genotypes, but also are superior genotypes in term of pomological characteristics such as nut and kernel weight, kernel percentage, shell thickness, kernel color, and quality. Most of the selected superior genotypes were protandrous, but genotype $\mathrm{FaBaCh} 2$ was protogynous. Protogyny is an important trait for genotypes as a pollinizer for commercial cultivars (Germain, 1990). Because of its direct impact on production, lateral bearing is considered as a main trait for the selection of walnut superior genotypes (Amiri et al., 2010). Lateral bearing of the studied superior genotypes was more than $50 \%$. Because most old Persian walnut genotypes in Iran have a terminal-bearing habit (Vahdati et al., 2014), special attention should be paid to improve this trait in future breeding programs.

The heritability of shell thickness, nut and kernel weight has been reported to be greater than 0.80 (Hansche et al., 1972). Therefore, these characteristic can be useful tools to select superior genotypes. Superior genotypes should have nut and kernel weight of more than 12 and $6 \mathrm{~g}$, respectively, kernel percentage greater than $50 \%$, L kernel color, thin-shell thickness (between 0.7 and 1.5 $\mathrm{mm}$ ), tight seal, and easily removable kernel from the shell (Akca and Ozongun, 2004; Khadivi-Khub et al., 2015). The genotypes selected and studied in the current study had shell thickness between 0.90 and $1.64 \mathrm{~mm}$, nut weight between 12.54 and $19.80 \mathrm{~g}$, kernel weight between 7.02 and $9.91 \mathrm{~g}$ and in general high kernel percentage, large nut size, and L to EL kernel color with easily removable shell and thereby performed better than previous reported genotypes (Arzani et al., 2008; Sharma and Sharma 2001a; Simsek et al., 2010).

PCA showed four groups within the selected genotypes. Late-leafing genotypes and genotypes with large genome size and nut weight were properly classified in separate groups. PCA and cluster analysis also showed that some genotypes from different regions clustered together into the same group. However, our primary phenotypic survey indicated a high genetic diversity in walnut populations of the Fars province in the southwest of Iran (Khadivi-Khub et al., 2015), and the clustering of the superior genotypes from different regions in the same group is presumably due to the short geographic distance between the two studied regions, and the use in the past of superior genotypes from the Bavanat region for development of walnut orchards in the Eqlid region.
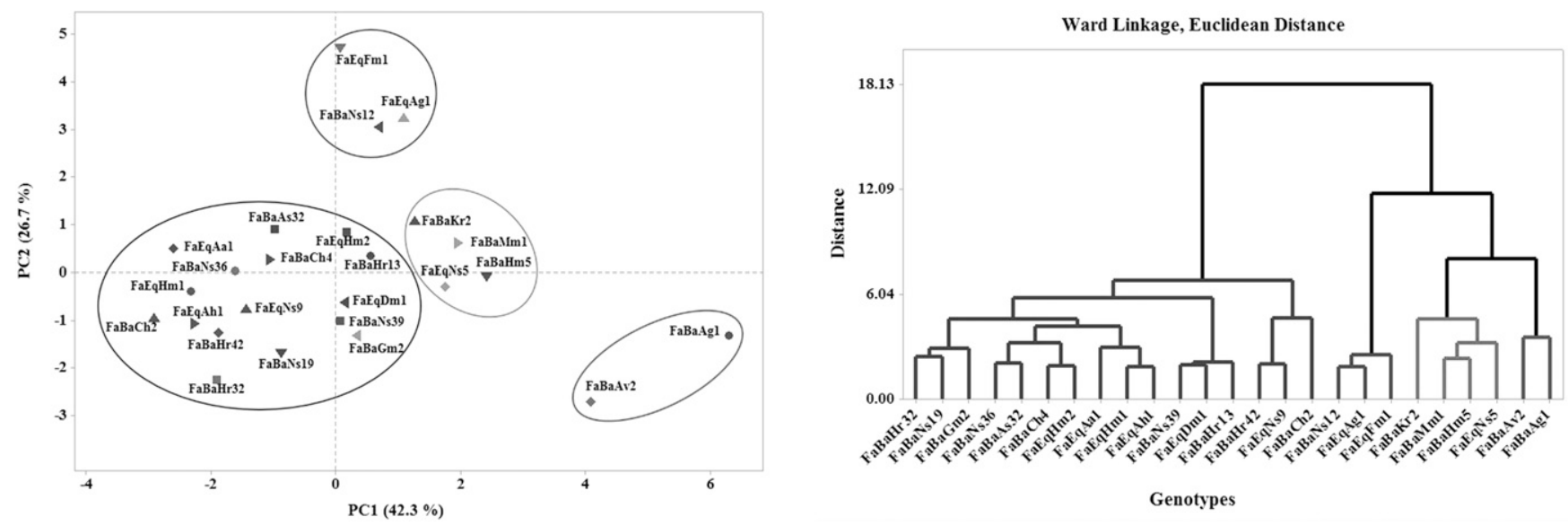

Fig. 2. Principal component analysis (left) and cluster analysis (right) of the studied walnut genotypes from southwest Iran based on phenotypic traits and genome size. 


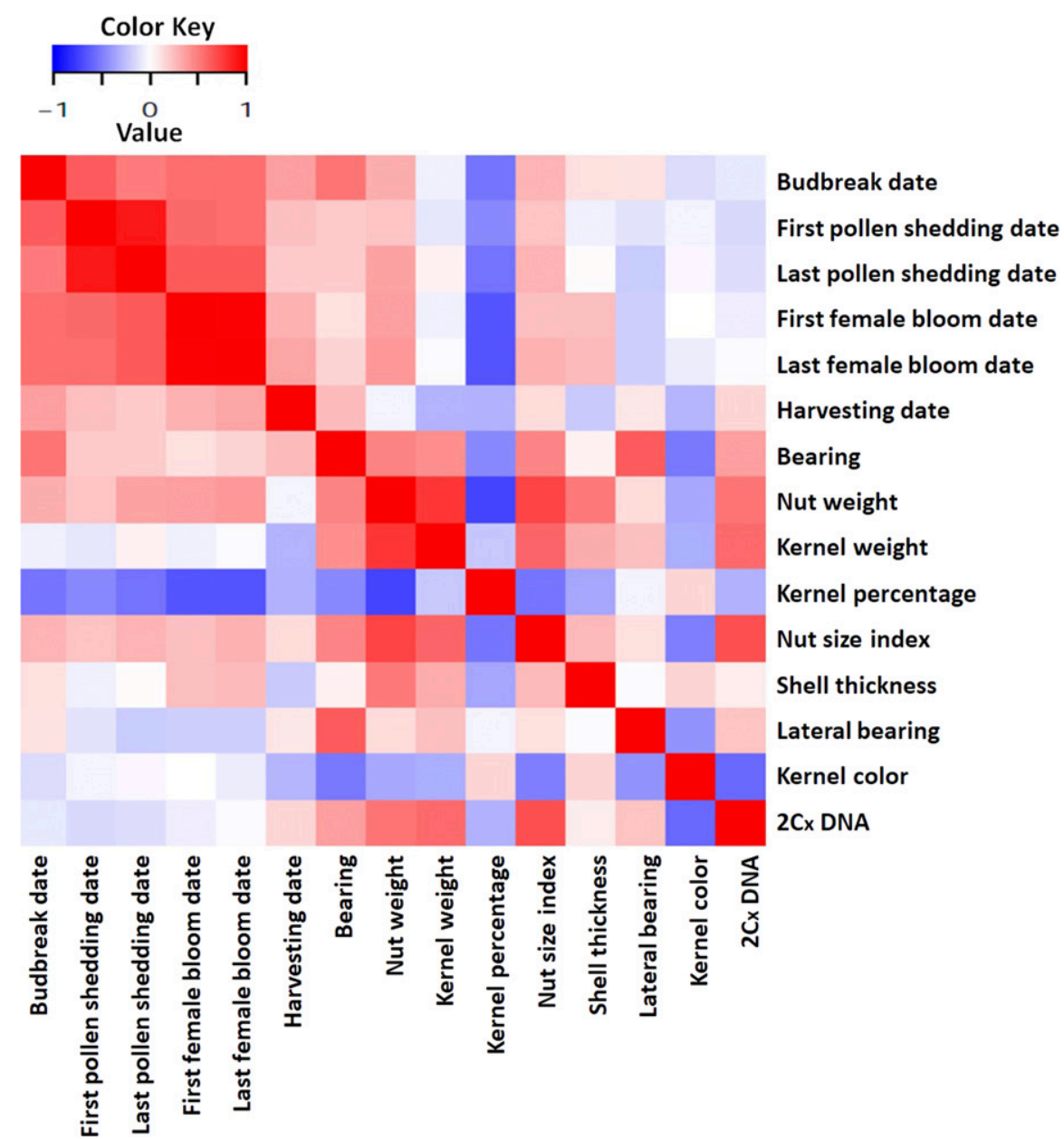

Fig. 3. Heat map of Spearman's correlations between genome size and phenotypic traits of the studied walnut superior genotypes from southwest Iran.

Fruit trees and especially walnut trees typically have long breeding cycles. Therefore, introducing new or improved cultivars by breeders may require many breeding cycles and dozens of years (van Nocker and Gardiner, 2014). Correlation studies can be useful tools to discover causal relationships between variables and can allow the prediction of future events from present knowledge. Therefore, correlation studies can be useful to reduce breeding cycles especially when the program is based on selection. Our results showed a positive correlation between different phenological characteristics, especially between leafing date (budbreak date) and harvesting date (Forde and McGranahan, 1996; Sarikhani Khorami et al., 2014). Furthermore, we found that lateral bearing, budbreak date, nut size, and nut weight have an effect on walnut production (yield) and that they were the main variables accounting for yield (Amiri et al., 2010; Solar et al., 2001). Because of their high heritability, walnut breeders are interested in pomological traits to identify superior genotypes. Therefore, finding correlations between pomological traits and other characteristics can be useful for the selection of superior genotypes (Amiri et al., 2010; Sarikhani Khorami et al., 2014). We found a significant and positive correlation size of superior genotypes in Eqlid was higher than for selected genotypes from the Bavanat region. In addition to genome size, average nut and kernel weight, and nut size of superior genotypes in Eqlid were higher than those from the Bavanat region. Interestingly, based on our results, there was a good positive correlation between genome size, nut and kernel weight, and nut size. We found a linear relation among genome size $(Y)$ with nut weight $\left(y=1.1642+0.012 x ; r=0.527^{* *}\right)$, kernel weight $(y=1.0746+0.0334 x$; $\left.r=0.551^{* *}\right)$, and NSI $(y=1.0275+0.0089 x$; $r=0.487^{* *}$ ). Previous studies also indicated that there was significant correlation between genome size and some phenotypic traits as well as geographic locations in various plant species, including Diphasiastrum sp. (Hanusova et al., 2014), Lathyrus sativus (Karimzadeh et al., 2011), and Iranian endemic muskmelon (Karimzadeh et al., 2010). Beaulieu et al. (2008) reported that genome size had a strong and significant correlation with cell size and stomatal density in 101 species of angiosperms. Based on our results, genome size can be a strong and valuable predictor of nut and kernel weight and nut size of walnut genotypes and thereby of total yield.

\section{Conclusion}

In conclusion, we found that there is a high genetic diversity in walnut population collected from southwest of Iran. The aim of walnut-breeding programs is high-yielding cultivars with some desirable characteristics such as late leafing, lateral bearing, large nut size, high kernel weight and percentage, and high kernel quality. Based on these characteristics, we selected 25 walnut superior genotypes out of 560 genotypes having many of these desirable characteristics such as high yield, late leafing, lateral bearing, and large nut and high kernel percentage. Therefore, these superior genotypes not only can be considered as gene bank for future breeding programs, but also have the potential to be introduced as commercial cultivars. We found some valuable correlations that can guide appropriate selection schemes for walnut-breeding programs. In addition, our results show that lateral bearing, budbreak date, nut size, and nut weight were the main variables to determine walnut production. Additionally, we evaluated genome size of different walnut superior genotypes for the first time in the world that could be very important for future breeding programs. There was a significant difference among walnut superior genotypes in term of $2 C_{x}$ DNA amount. These results were additional evidence of high genetic diversity of the walnut population in southwest Iran. Furthermore, we found that all superior genotypes were diploid $(2 n=2 x=32)$ and $2 C_{x}$ DNA amount varied from 1.29 to $1.42 \mathrm{pg}$ (corresponding to 1261.62 till $1388.76 \mathrm{Mbp}$ ). Based on the results, human genome size (6153 Mbp) is $\approx 4.7$ times of walnut genome size. A linear relation was found among genome size and nut and kernel weight, and 

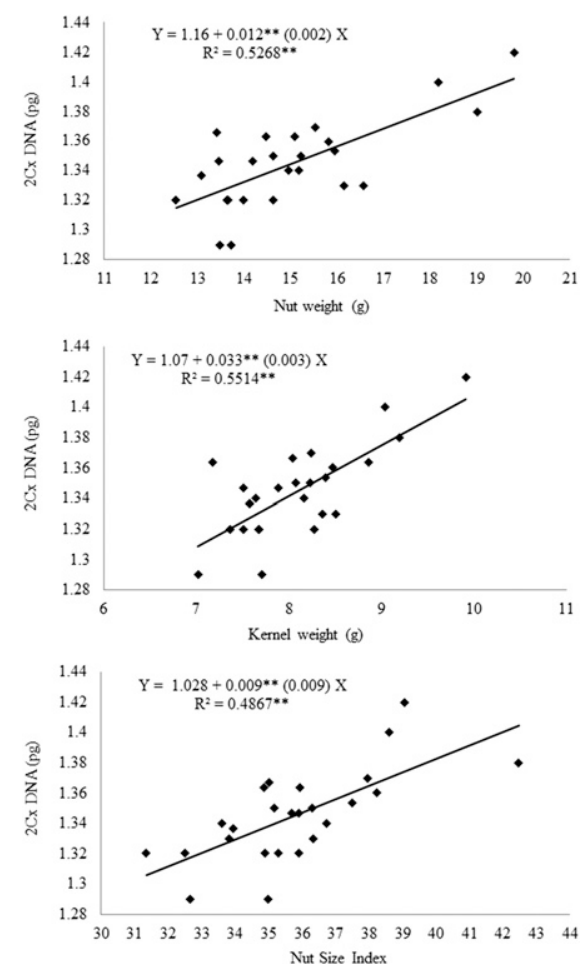

Fig. 4. Linear regression between genome size $\left(2 C_{x}\right.$ DNA) and nut weight, kernel weight and nutsize index (SI) of the studied walnut superior genotypes from southwest Iran.

NSI. Generally, genome size can be considered as a strong and a valuable predictor of nut and kernel weight and nut size of walnut genotypes, although further research is needed to confirm this relation beyond the genotypes of southwest of Iran.

\section{Literature Cited}

Abedi, R., A. Babaei, and G. Karimzadeh. 2015. Karyological and flow cytometric studies of Tulipa (Liliaceae) species from Iran. Plant Syst. Evol. 301(5):1473-1484.

Akca, Y. and S. Ozongun. 2004. Selection of late leafing, late flowering, laterally fruitful walnut (Juglans regia) types in Turkey. N. Z. J. Crop Hort. Sci. 32(4):337-342.

Amiri, R., K. Vahdati, S. Mohsenipoor, M.R. Mozaffari, and C. Leslie. 2010. Correlations between some horticultural traits in walnut. HortScience 45:1690-1694.

Arzani, K., H. Mansouri Ardakan, A. Vezvaei, and M.R. Roozban. 2008. Morphological variation among Persian walnut (Juglans regia) genotypes from central Iran. N. Z. J. Crop Hort. Sci. 36:159-168.

Aslantas, R. 2006. Identification of superior walnut (Juglans regia) genotypes in north-eastern Anatolia, Turkey. N. Z. J. Crop Hort. Sci. 34:231-237.

Baird, W.V., A.S. Estager, and J.K. Wells. 1994. Estimating nuclear DNA content in peach and related diploid species using laser flow cytometry and DNA hybridization. J. Amer. Soc. Hort. Sci. 119(6):1312-1316.

Beaulieu, J.M., I.J. Leitch, S. Patel, A. Pendharkar, and C.A. Knight. 2008. Genome size is a strong predictor of cell size and stomatal density in angiosperms. New Phytol. 179:975-986.
Bernard, A., F. Lheureux, and E. Dirlewanger. 2018. Walnut: Past and future of genetic improvement. Tree Genet. Genomes 14(1):128.

Bhat, T.M. and R. Kudesia. 2011. Evaluation of genetic diversity in five different species of family solanaceae using cytological characters and protein profiling. Genet. Eng. Biotechnol. J. 20:20-25.

Brito, G., J. Loureiro, T. Lopes, E. Rodriguez, and C. Santos. 2008. Genetic characterization of olive trees from Madeira Archipelago using flow cytometry and microsatellite markers. Genet. Resources Crop Evol. 55(5):657-664.

Doležel, J. and J. Bartos. 2005. Plant DNA flow cytometry and estimation of nuclear genome size. Ann. Bot. 95:99-110.

Doležel, J., J. Bartos, H. Voglmayr, and J. Greilhuber. 2003. Nuclear DNA content and genome size of trout and human. Cytometry A 51:127-129.

Doležel, J., J. Greilhuber, and J. Suda. 2007. Estimation of nuclear DNA content in plants using flow cytometry. Nat. Protoc. 2:22332244.

Ebrahimi, A., A. Khadivi-Khub, Z. Nosrati, and R. Karimi. 2015. Identification of superior walnut (Juglans regia) genotypes with late leafing and high kernel quality in Iran. Scientia Hort. 193:195-201.

FAO. 2014. FAOSTAT production crops. $16 \mathrm{Mar}$. 2014. <http://www.fao.org/faostat/en/\#data/QC>.

Forde, H.I. and G.H. McGranahan. 1996. Walnuts, p. 241-273. In: J. Janick and J.N. Moore (eds.). Fruit Breeding, Volume III: Nuts. John Wiley and Sons, Inc., New York, NY.

Freitas, N.M., T.N. Pereira, I.G. Geronimo, A.O Azevedo, S.R. Ramos, and M.G. Pereira. 2015. Coconut genome size determined by flow cytometry: Tall versus Dwarf types. Genet. Mol. Res. 15(1):1-9.

Germain, E. 1990. Inheritance of late leafing and lateral bud fruitfulness in walnut (Juglans regia L.) phenotypic correlations among some traits of the trees. Acta Hort. 284:125-134.

Ghasemi, M., K. Arzani, and D. Hassani. 2012. Evaluation and identification of walnut (Juglans regia L.) genotypes in Markazi province of Iran. Crop Breeding J. 2(2):119-124.

Govindaraj, M., M. Vetriventhan, and M. Srinivasan. 2015. Importance of genetic diversity assessment in crop plants and its recent advances: An overview of its analytical perspectives. Genet. Res. Intl. 2015:1-14, doi: 10.1155/2015/431487.

Greilhuber, J., J. Doležel, M.A. Lysák, and M.D. Bennett. 2005. The origin, evolution and proposed stabilization of the terms 'genome size' and 'C-value' to describe nuclear DNA contents. Ann. Bot. 95:255-260.

Hansche, P.E., V. Beres, and H.I. Forde. 1972. Estimates of quantitative genetic properties of walnut and their implications for cultivar improvement. J. Amer. Soc. Hort. Sci. 97:279285.

Hanusova, K., L. Ekrt, P. Vít, F. Kolář, and T. Urfus. 2014. Continuous morphological variation correlated with genome size indicates frequent introgressive hybridization among Diphasiastrum species (Lycopodiaceae) in central Europe. PLoS One 9(6):1-13.

Hofer, M. and A. Meister. 2010. Genome size variation in Malus species. J. Bot. 2010:1-8.

International Human Genome Sequencing Consortium. 2004. Finishing the euchromatic sequence of the human genome. Nature 431 (7011):931-945.

IPGRI. 1994. Descriptors for walnut (Juglans spp.). Intl. Plant Genet. Resources Inst., Rome, Italy.
Javadian, N., G. Karimzadeh, M. Sharifi, A. Moieni, and M. Behmanesh. 2017. In vitro polyploidy induction: Changes in morphology, podophyllotoxin biosynthesis, and expression of the related genes in Linum album (Linaceae). Planta 245(6):1165-1178.

Karimi, R., A. Ershadi, K. Vahdati, and K. Woeste. 2010. Molecular characterization of Persian walnut populations in Iran with microsatellite markers. HortScience 45:1403-1406.

Karimzadeh, G., M. Danesh-Gilevaei, and M. Aghaalikhani. 2011. Karyotypic and nuclear DNA variations in Lathyrus sativus (Fabaceae). Caryologia 64(1):42-54.

Karimzadeh, G., S.H. Mousavi, M. JafarkhaniKermani, and M. Jalali-Javaran. 2010. Karyological and nuclear DNA variation in Iranian endemic muskmelon (Cucumis melo var. inodorus). Cytologia (Tokyo) 75(4):451-461.

Keles, H., Y. Akca, and S. Ercisli. 2014. Selection of promising walnut genotypes (Juglans regia L.) from inner Anatolia. Acta Sci. Pol. Hortoru. 13(3):167-173.

Khadivi-Khub, A. and A. Ebrahimi. 2015. The variability in walnut (Juglans regia L.) germplasm from different regions in Iran. Acta Physiol. Plant. 37(3):1-11.

Khadivi-Khub, A., A. Ebrahimi, A. Mohammadi, and A. Kari. 2015. Characterization and selection of walnut (Juglans regia L.) genotypes from seedling origin trees. Tree Genet. Genomes 11(3):1-10.

Knight, C.A., N.A. Molinari, and D.A. Petrov. 2005. The large genome constraint hypothesis: Evolution, ecology and phenotype. Ann. Bot. 95:177-190.

Korban, S.S., W. Wannarat, C.M. Rayburn, T.C. Tatum, and A.L. Rayburn. 2009. Genome size and nucleotypic variation in Malus germplasm. Genome 52(2):148-155.

Loureiro, J., E. Rodriguez, A. Costa, and C. Santos. 2007b. Nuclear DNA content estimations in wild olive (Olea europaea L. ssp. europaea var. sylvestris Brot.) and Portuguese cultivars of $O$. europaea using flow cytometry. Genet. Resources Crop Evol. 54(1):21-25.

Loureiro, J., E. Rodriguez, J. Doležel, and C. Santos. 2007a. Two new nuclear isolation buffers for plant DNA flow cytometry: A test with 37 species. Ann. Bot. 100:875-888.

Ma, J. and C.I. Amos. 2012. Principal components analysis of population admixture. PLoS One. 7(7):40115.

Mahdavi, S. and G. Karimzadeh. 2010. Karyological and nuclear DNA content variation in some Iranian endemic Thymus species (Lamiaceae). J. Agr. Sci. Technol. 12:447-458.

Majdi, M., G. Karimzadeh, M.A. Malboobi, R. Omidbaigi, and G. Mirzaghaderi. 2010. Induction of tetraploidy to feverfew (Tanacetum parthenium Schulz-Bip.): Morphological, physiological, cytological and phytochemical changes. HortScience 45(1):16-21.

Podwyszyńska, M., D. Kruczyńska, A. Machlańska, B. Dyki, and I. Sowik. 2016. Nuclear DNA content and ploidy level of apple cultivars including polish ones in relation to some morphological traits. Acta Biologica Cracoviensia s. Botanica. 58(1):81-93.

Petrov, D.A. 2001. Evolution of genome size: New approaches to an old problem. Trends Genet. 17(1):23-28.

Ramos, D.E. 1997. Walnut production manual. Vol. 3373. UCANR Publication, University of California.

Sadat Hosseini Grouh, M., K. Vahdati, M. Lotfi, D. Hasani, and N. Pirvali Biranvand. 2011. Production of haploids in Persian walnut through 
parthenogenesis induced by gamma-irradiated pollen. J. Amer. Soc. Hort. Sci. 136(3):198204.

Sadat Noori, S.A., M. Norouzi, G. Karimzadeh, K.H. Shirkool, and M. Niazian. 2017. Effect of colchicine-induced polyploidy on morphological characteristics and essential oil composition of ajowan (Trachyspermum ammi L.). Plant Cell Tissue Organ Cult. 130:543-551.

Sarikhani Khorami, S., K. Arzani, and M.R. Roozban. 2014. Correlations of certain high-heritability horticultural traits in Persian walnut (Juglans regia L.). Acta Hort. 1050:61-68.

Sharma, S.D. and O.C. Sharma. 2001a. Genetic divergence in seedling trees of Persian walnut (Juglans regia L.) for various metric nut and kernel characters in Himachal Pradesh. Scientia Hort. 88:163-171.

Sharma, S.H. and O.C. Sharma. 2001b. Studies on variation in nut and kernel characters and selection of superior walnut seedlings (Juglans regia 1.) from Garsa and Jogindernagar areas of Himachal Pradesh. Acta Hort. 544:47-50.
Simsek, M., K.U. Yilmaz, and A.R. Demirkiran. 2010. Selection and determination of some significant properties of superior walnut genotypes. Sci. Res. Essays 5(19):2987-2996.

Solar, A., M. Hudina, and F. Stampar. 2001. Relationship between tree architecture, phenological data and generative development in walnut (Juglans regia L.). Acta Hort. 544:275-286.

Suda, J., L.A. Meyerson, I.J. Leitch, and P. Pyšek. 2015. The hidden side of plant invasions: The role of genome size. New Phytol. 205(3):994 1007.

Swift, H. 1950. The constancy of deoxyribose nucleic acid in plant nuclei. Proc. Natl. Acad. Sci. USA 36(11):643-654.

Tarkesh Esfahani, S., G. Karimzadeh, and M.R. Naghavi. 2016. 2C DNA value of Persian poppy (Papaver bracteatum Lindl.) medicinal plant as revealed by flow cytometry analysis; a quick effective criteria for distinguishing unidentified papaver species. Intl. J. Adv. Biotechnol Res. 7(2):573-578.
Tavan, M., M.H. Mirjalili, and G. Karimzadeh. 2015. In vitro polyploidy induction: Changes in morphological, anatomical and phytochemical characteristics of Thymus persicus (Lamiaceae) Plant Cell Tissue Organ Cult. 122(3):573-583.

Vahdati, K., D. Hassani, R. Rezaee, M.H. Jafari Sayadi, and S. Sarikhani Khorami. 2014. Following walnut footprints (Juglans regia L.) cultivation and culture, folklore and history, traditions and uses; Chapter 24: Walnut footprint in Iran. Scripta Hort. 17:187-201.

van Nocker, S. and S.E. Gardiner. 2014. Breeding better cultivars, faster: Applications of new technologies for the rapid deployment of superior horticultural tree crops. Hort. Res. $1: 14022$.

Yarilgac, T., F. Koyuncu, M.A. Koyuncu, A. Kazankaya, and S.M. Sen. 2001. Some promising walnut selections (Juglans regia L.). Acta Hort. 544:93-100.

Zeneli, G., H. Kola, and M. Dida. 2005. Phenotypic variation in native walnut populations of Northern Albania. Scientia Hort. 105:91-100. 